Boise State University

ScholarWorks

Educational Technology Faculty Publications and

Presentations

Department of Educational Technology

$1-1-2012$

Empowering Educators with Google's Android App Inventor: An Online Workshop in Mobile App Design

Yu-Chang Hsu

Boise State University

Kerry Rice

Boise State University

Lisa Dawley

Boise State University 


\title{
Empowering educators with Google’s Android App Inventor: An online workshop in mobile app design
}

\author{
Yu-Chang Hsu, Kerry Rice and Lisa Dawley
}

Address for correspondence: Dr Yu-Chang Hsu, Assistant Professor, Department of Educational Technology, Boise State University, 1910 University Drive, Boise, ID 83725-1747, USA. Email: hsu@boisestate.edu

\section{Introduction}

Mobile devices such as smartphones are vastly gaining popularity (Johnson, Levine, Smith, \& Stone, 2010) due to their relatively strong computing capability built into small sizes, their Internet connectivity, and the availability of various types and easy-to-use mobile software applications ("mobile apps"). It is estimated that by 2015, 80\% of people accessing the Internet will do it through cell phones (Johnson, Smith, Willis, Levine, \& Haywood, 2011). Mobile technologies are now gaining increased attention and popularity across education sectors, which has led to innovation in mobile app design (Johnson et al., 2010).

Android OS has now caught up and led the mobile OS market within the U.S. with 38.1\% share (Haselton, 2011). Android OS has the advantage of being open-source, customizable and allows for multitasking. As of July 2011, there were 250,000 Android apps on the Android Market, with a total of 4.5 billion downloads (Wikipedia, 2011a). With the public release of App Inventor, building mobile apps without prior coding experiences is now a possibility.

In this report, the authors describe an introductory-level mobile app design workshop developed and offered over six weeks in Summer 2011. We also discuss the challenges and instructional implications derived from our experiences with this workshop.

\section{What is App Inventor?}

App Inventor (AI) is a web-based tool consisted of two major elements-Component Designer and Block Editor, which together allow users without prior programming experience to develop mobile apps (Wikipedia, 2011b). Through Component Designer, one can design the app's interface and integrate the non-visible components (e.g., Global Positioning System (GPS) or sound). With Block Editor, one can program apps' behaviors and tell how an app reacts under certain circumstances. The most powerful part of AI lies in its visual programming capability, where one can drag, drop, and arrange various interfaces, non-visible components, and behavior logic blocks to create full functioning mobile apps.

\section{App design workshop and online learning environments Context and learning environments}

This introductory-level mobile app design workshop was offered through Boise State University's Professional Development (PD) Portal, hosted on the Moodle learning management system (provided by Moodlerooms, Inc. based in Baltimore, MD, USA). The purpose of the workshop was to help participants leverage the potential of mobile computing technologies to assist them in their teaching, learning, and work. In this workshop, participants communicated about mobile learning and favorite apps through Twitter by including a designated workshop hashtag (\#BSUAppPD). In addition to the instruction on learning activities hosted on the PD portal, the instructor created a course wiki using Google Sites for workshop participants to share their apps for peer review and provided constructive feedback through a "comment" function (see Figure 1 for a snapshot of the wiki site). 


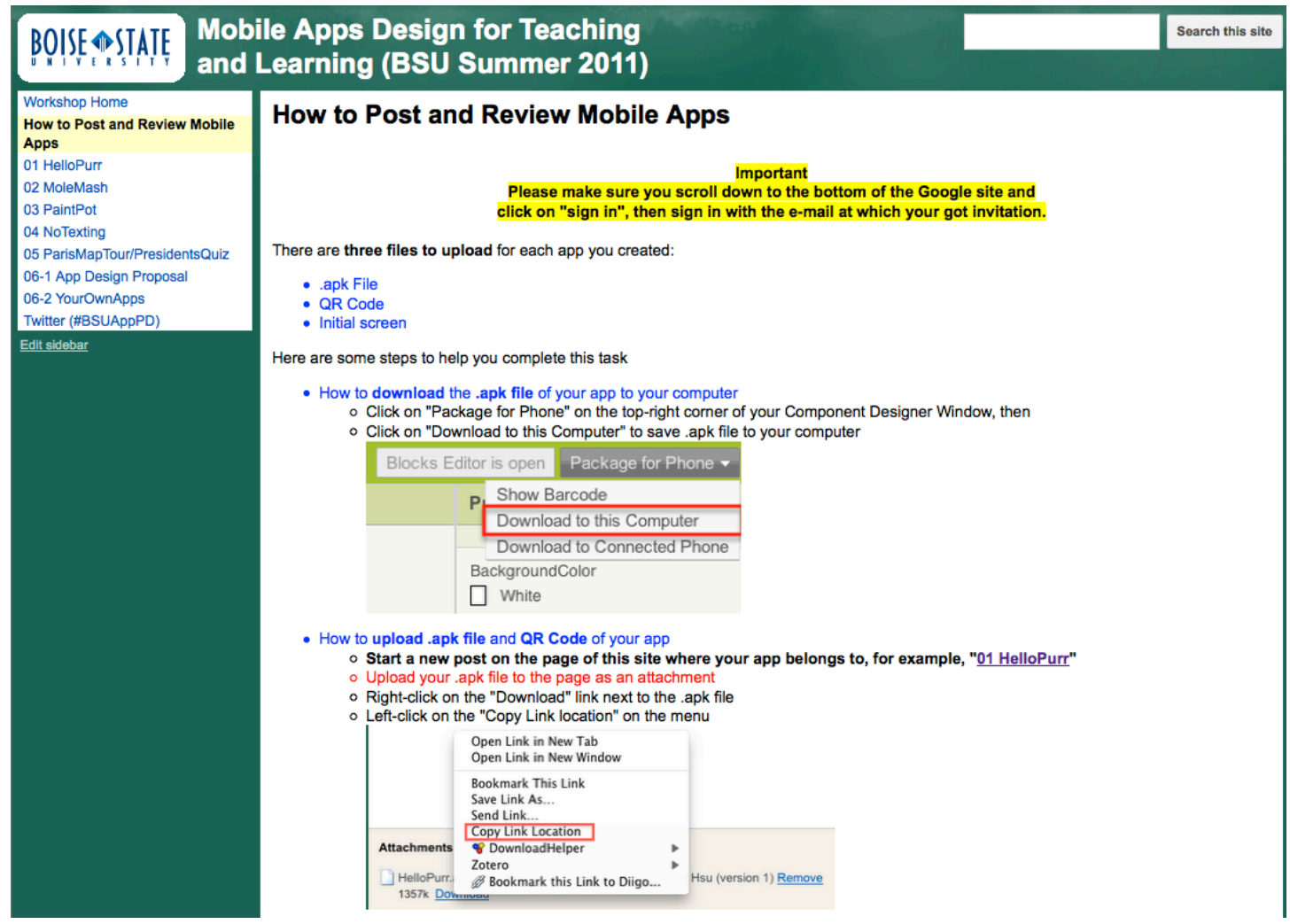

Figure 1: A sample instruction on posting and reviewing mobile apps on the workshop wiki

The learning environments, including AI, complemented each other to help form a virtual learning community of mobile app design (see Figure 2).

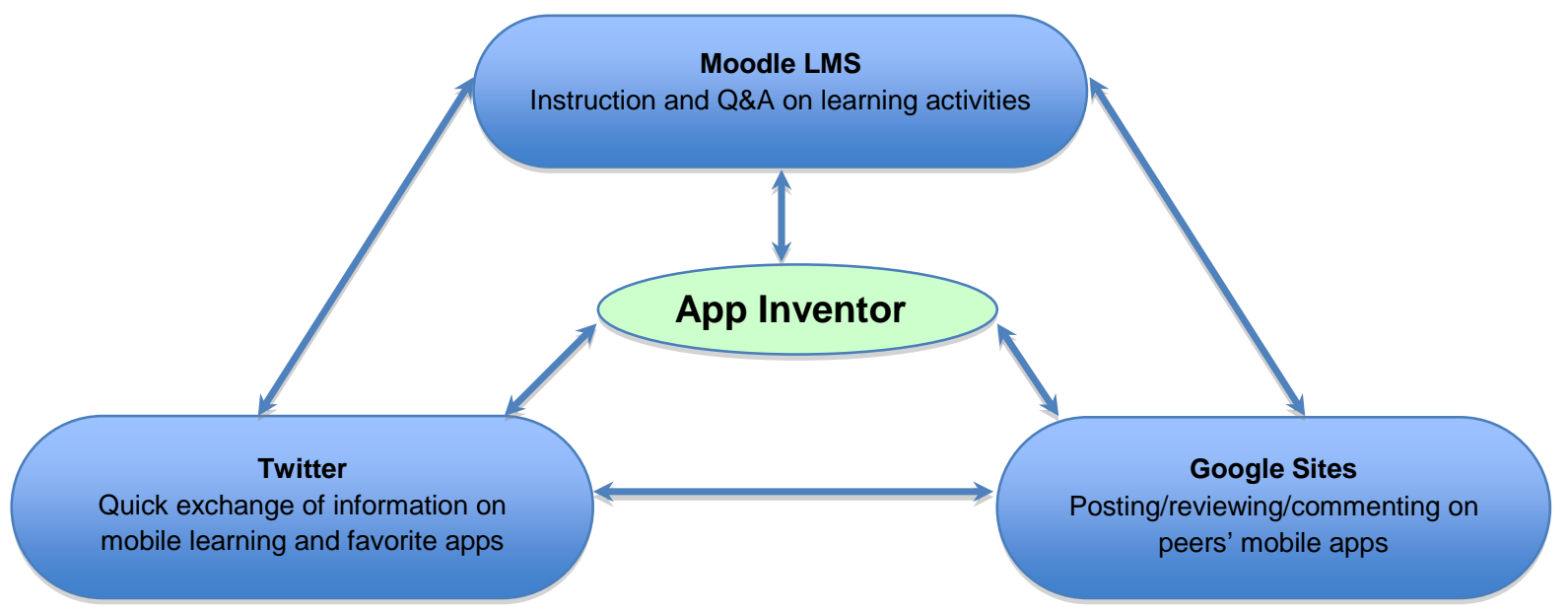

Figure 2: The integration of learning environments in the online workshop in mobile app design 


\section{Participants}

There were five enrolled participants, and four among the five were active throughout the workshop. The small enrollment turned out to be beneficial for the participants because the members were able to provide sufficient constructive feedback for their peers and to help test their peers' mobile apps.

\section{Workshop structure, facilitation, activities}

The workshop consisted of three modules, lasting two weeks each. Participants were expected to expend 15 hours of effort in each module. Throughout the workshop, the instructor communicated with participants mostly through module instruction, weekly announcements in the LMS (Learning Management System), Twitter, and e-mail.

- Module 1: Introduction to Mobile Computing and Android App Inventor. Designed to help participants learn about the potential of mobile apps in education, to evaluate and share one's favorite apps, and to create a virtual learning community for mobile app design. This module introduced participants to the mobile apps knowledge base, the concept of interactive design, and the major categories of mobile apps (i.e., education, game, productivity etc.).

- Module 2: Hands-on with App Inventor. Participants learned about basic components for building apps, and built practice apps by following tutorials in the textbook authored by Wolber, Abelson, Spertus, and Looney (2011). During the process, the instructor provided suggestions and facilitated discussion on block programming. Each participant was asked to post their .apk files (executable file on Android devices), screenshots of their apps, and QR (Quick Response Code) codes for others to download their apps. The participants also actively reviewed, provided feedback, and engaged in conversation on peers' practice apps, app design proposals, and final project apps.

- Module 3: Design with App Inventor. Participants built one last practice app, proposed an app of their own, and built a working prototype or completed app. In addition, participants continuously engaged in active app review-feedback process similar to that in Module 2.

\section{Practice app design and customization}

The following practice apps and corresponding apps were covered in this workshop:

1. HelloPurr: A simple app in which participants get familiar with the AI work environment (i.e., Component Designer and Block Editor). The app delivers a cat meow sound when a button is touched.

2. PaintPot: Allows users to draw lines and dots of different colors with fingers through a smartphone touchscreen. Users program the app's memory so that the phone remembers the color chosen by the users for making their drawing on the screen.

3. MoleMash: A game-based app where players touch the randomly appearing moles to score points. This app introduces programming of animated images (called "sprite” in AI) and random integers.

4. NoTextingWhileDriving: Users respond to texting without actually holding their phones and typing. It sends automatic predefined response messages. Students are introduced to programming with the texting and database (called “TinyDB” in AI) components.

5. ParisMapTour or Presidents Quiz: In ParisMapTour users select from a list of place names and link to Google Map to view the street photos, while the Presidents Quiz allows users to take a trivia quiz about U.S. presidents through a linearly sequenced list. Both apps require students to program "lists of data” for user selection.

For each of the practice mobile app assignments, the participants were asked to "change at least one aspect" of their apps to show customization. Participants started with replacing generic images (e.g., the cat in HelloPurr app) with the images of their own pets. As they developed skills, they began to add more advanced customizations. For example, in MoleMash, one student added an animated image component to take away points once that image was touched/hit, in addition to the original scoring-only game rule. In Presidents Quiz, one of the students changed the short-text version of text into multiple-choice questions. The advancement of participants' customizations demonstrated an increasing comfort with using AI to build apps. 


\section{Design proposal and final project apps}

Participants were required to submit their final app design proposals for peer and instructor review prior to building the apps. During this process, they were prompted to think through different aspects of design such as visual interface and the level of interactivity. Participants were also guided to map out the visible and non-visible "behaviors" their apps would execute. Participants built their apps and were encouraged to revisit/revise their proposals as their app design progressed.

Among the four final apps designed by workshop participants, two were customized quiz-type apps that served the learning needs of their individual settings—one was designed to help primary school students review stories they read, while another helped chiropractic students review for board exams. Another student created an app on geography to help determine the exact opposite location on earth from where the user stands. The last student developed a proposal to create a weather app that presented key weather information for one's choice of Top 10 places. Although a working prototype was not completed due to time constraints and the lack of more advanced skills in app design, this student presented a workable plan.

\section{Challenges and implications}

While students commented on enjoying this class and testing other people's apps, there were some challenges The QR codes submitted by students sometimes returned a page with a link to an app's file instead of directly downloading the file to one's phone. This should not be a problem when Google Sites service is more stable. Instructors can also have participants use other similar wiki systems that allow multiple users to add content. Another solution is to use a different web-based QR code generator.

The three-module format and six-week length seemed to work well for an introductory workshop on mobile app design. However, since the first few practice apps are relatively simple, it might make sense to reduce the time for required readings and discussions, and plan for participants to do hands-on design during the second week of Module 1. This way, participants would be engaged in development earlier in the process which may increase motivation and interest. Two other benefits include: 1) participants can build more practice apps and gain more block programming skills to be applied in their final app design; 2) the shorter turn-around on the Twitter activity for discussing mobile learning and mobile computing could lead to more focused and active participation.

\section{Conclusion}

Overall, this workshop served the purpose of introducing visual programming of mobile apps to interested educators and individuals. It also helped participants realize the potential of mobile computing and mobile apps in their teaching, learning, and work settings. Most important of all, this workshop helped participants realize they can do it-leverage the power of mobile computing, and design their own apps to serve their needs.

\section{References}

Haselton, T. (2011). Android market share jumps as BlackBerry slides; smartphones now owned by 1 in 3 Americans. Retrieved September 22, 2011 from http://www.bgr.com/2011/07/05/android-market-share-jumpsas-blackberry-slides-smartphones-now-owned-by-1-in-3-americans/

Johnson, L., Levine, A., Smith, R., \& Stone, S. (2010). The 2010 Horizon Report. Austin, TX: The New Media Consortium.

Johnson, L., Smith, R., Willis, H., Levine, A., and Haywood, K., (2011). The 2011 Horizon Report. Austin, TX: The New Media Consortium.

Wikipedia (2011a). Android Market. Retrieved September 22, 2011 from http://en.wikipedia.org/wiki/Android_Market

Wikipedia (2011b). Google App Inventor. Retrieved September 22, 2011 from http://en.wikipedia.org/wiki/Google_App_Inventor

Wolber, D., Abelson, H., Spertus, E., \& Looney. L. (2011). App Inventor: Create Your Own Android Apps (1st ed.). Sebastopol, CA: O'Reilly Media. 


\section{To cite this paper}

Hsu, Y., Rice, K., \& Dawley, L. (2012). Empowering educators with Google's Android App Inventor: An online workshop in mobile app design. British Journal of Educational Technology, 43(1), E1-E5. doi:10.1111/j.14678535.2011.01241.x 